IMPACT OF NEUROSENSORIAL DISORDERS ON MOOD IN OLDER PEOPLE

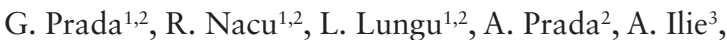
I. Alexa ${ }^{3}, \mathrm{~A}$. Herghelegiu ${ }^{2}, 1$. Clinical department, National Institute of Gerontology and Geriatrics "Ana Aslan", BUCHAREST, Romania, 2. University of Medicine and Pharmacy "Carol Davila”, Bucharest, Romania, 3. "Gr.T. Popa" University of Medicine and Pharmacy, Bucharest, Romania

Introduction

Prevalence of neurosensorial disorders increases with age, having multiple impact in elderly, including on mood. Study objective was to identify effects of neurosensorial disorders on mood in elderly, including their relationship with comorbidities.

Material and Methods

A total of 304 subjects, age-range 50-95 years, were included, divided into two groups: 152 adults (50-64 years) and 152 elderly (75-95 years).

Results

Visual impairments had highest prevalence in elderly, retinopathy being main cause. Glaucoma was more prevalent in women $(\mathrm{p}<0.01)$, irrespective of age $(\mathrm{p}>0.05)$ and it correlated with mood disorders $(\mathrm{r}=0.87)$. Diabetic retinopathy, most prevalent visual disorders in men and correlated with mood disorders $(r=0.67)$. Depression severity decreased with age, presumably due to an adaptation effect. Other visual disorder: cataract, significantly more prevalent in older women $(p<0.01)$. It correlated with mood disorders $(r=0.73)$. Hearing disorders were significantly less prevalent in both groups and had less impact on mood disorders in elderly $(\mathrm{r}=0.51)$. Comorbidities have been identified. Rheumatic diseases were most prevalent in elderly, followed by diabetes mellitus and cardiovascular disorders. Rheumatic disorders had more important impact on mood in elderly $(\mathrm{p}<0.01)$, irrespective of gender $(p>0.05)$, and the effect increased when corroborated with neurosensorial disorders. This effect was less prominent in adults. Cardiovascular diseases had less effect on mood, being correlated mainly with mild depression $(\mathrm{r}=0.59)$. Concomitant presence of neurosensorial disorders with somatic conditions reduces therapeutic compliance in elderly.

Conclusions

Comorbidities have a significant impact on mood disorders in elderly with neurosensorial disorders. Consequently the three conditions should be addressed concomitantly.

\section{ARE HIV-INFECTED OLDER ADULTS AGING DIFFERENTLY?}

S. Karpiak ${ }^{1,2}$, R.J. Havlik ${ }^{1}$, A. Ambroziak ${ }^{1,3}, 1$. ACRIA Center on HIV \& Aging, New York, New York, 2. New York University College of Nursing, New York, New York, 3. Warsaw University, Warsaw, Poland

Older adults with HIV are experiencing non-HIV related comorbidities. We evaluated the onset and frequency of multimorbidity in HIV infected older adults derived from a New York City cohort (N=914) ROAH (Research on Older Adults with HIV). ROAH data was compared, after matching, to samples from the National Health and Nutrition Examination Surveys (NHANES \& NYCNHANES). Comparisons were based on self-reported conditions (arthritis, cancer, depression, diabetes, CVD, hypertension, respiratory condition, stroke, HIV). When assessing the number of comorbid conditions $(0-1,2,3,4,5,6+)$, older adults wirth HIV evidenced averages of $2.65 / 2.60$ comorbidities, significantly higher than NHANES (1.65/1.48). Depression was the highest frequency in ROAH (52 \& 62\%) vs NHANES (17 \& 21\%). In multivariate logistic models, depression was best explained by younger age, HIV, morbidity number, unemployment. Analyses do not support hypotheses of accelerated aging. Increasingly the need for HIV care providers to embrace geriatric care principles optimized to manage multimorbidity and contributing risks emerges.

\section{SESSION 4345 (PAPER)}

\section{SUCCESSFUL AGING: CROSS-NATIONAL AND LONGITUDINAL PERSPECTIVES}

\section{TRANSITIONING FROM SUCCESSFUL AGING: LONGITUDINAL ANALYSES OF PREDICTORS AND PATTERNS}

T.M. Cooney ${ }^{1}$, A.L. Curl ${ }^{2}$, 1. Sociology, University of Colorado--Denver, Denver, Colorado, 2. Department of Family Science \& Social Work, Miami University, Oxford, Obio

Despite extensive research using Rowe and Kahn's model of successful aging, its application to representative national samples is rare. Longitudinal research permitting analysis of transitions to non-successful aging (NSA) over time at the individual level also is lacking. We apply event history analysis to 8 waves (2-year intervals from 1998-2012) of representative data for 11,346 individuals ages 50 and older from the Health and Retirement Study in the U.S. to examine influences on the transition into NSA. We extend prior research by considering the impact of factors present at birth (e.g., race, place of birth), as well as time-varying adult statuses (e.g, education, income) and lifestyle factors (e.g., smoking). Significant predictors of NSA include age and parents' longevity. Though the results also reveal that NSA is accelerated for Blacks, Hispanics, females, and those born in the Southern U.S., both race and ethnicity become insignificant once adult status factors and lifestyle variables are considered. High BMI and smoking positively influence transitions to NSA, while higher education and income delay NSA. Also examined are components of successful aging that are most often implicated in NSA transitions at distinct ages. For persons transitioning to NSA between ages 50 and 69, mobility problems and chronic disease are the most common NSA dimensions reported. But, for persons transitioning to NSA from age 70 on, lack of productive engagement is the most common dimension, followed by mobility problems and chronic illness. Implications of all of these findings for interventions promoting successful aging are addressed. 